\title{
Shopping Context and Consumers' Mental Representation of Complex Shopping Trip Decision Problems
}

\author{
Benedict G.C. Dellaert, Theo A. Arentze and Harry J.P. Timmermans
}

\begin{tabular}{|l|l|}
\hline \multicolumn{2}{|l|}{ ERIM REPORT SERIES RESEARCH IN MANAGEMENT } \\
\hline ERIM Report Series reference number & ERS-2008-016-MKT \\
\hline Publication & March 2008 \\
\hline Number of pages & 47 \\
\hline Persistent paper URL & http://hdl.handle.net/1765/11812 \\
\hline Email address corresponding author & dellaert@few.eur.nl \\
\hline Address & Erasmus Research Institute of Management (ERIM) \\
& RSM Erasmus University / Erasmus School of Economics \\
& Erasmus Universiteit Rotterdam \\
& P.O.Box 1738 \\
& 3000 DR Rotterdam, The Netherlands \\
& Phone: $\quad+31104081182$ \\
& Fax: $\quad+31104089640$ \\
& Email: info@erim.eur.nl \\
& Internet: $\quad$ www.erim.eur.nl \\
\hline
\end{tabular}

Bibliographic data and classifications of all the ERIM reports are also available on the ERIM website: www.erim.eur.nl 


\section{ERASMUS RESEARCH INSTITUTE OF MANAGEMENT}

\section{REPORT SERIES}

\section{RESEARCH IN MANAGEMENT}

\begin{tabular}{|c|c|}
\hline \multicolumn{2}{|c|}{ ABSTRACT AND KEYWORDS } \\
\hline Abstract & $\begin{array}{l}\text { Depending on the shopping context, consumers may develop different mental representations of } \\
\text { complex shopping trip decision problems to help them interpret the decision situation that they } \\
\text { face and evaluate alternative courses of action. To investigate these mental representations and } \\
\text { how they vary across contexts, the authors propose a causal network structure that allows for a } \\
\text { formal representation of how context-specific benefits requirements affect consumers' evaluation } \\
\text { of decision alternative attributes. They empirically test hypotheses derived from the framework, } \\
\text { using data on consumers' mental representations of a complex shopping trip decision problem } \\
\text { across four shopping contexts that differ in terms of opening hour restrictions and shopping } \\
\text { purpose, and find support for the proposed structure and hypotheses. }\end{array}$ \\
\hline Free Keywords & $\begin{array}{l}\text { retailing, consumer decision-making, mental representations, context effects, } \\
\text { shopping trip decisions }\end{array}$ \\
\hline Availability & $\begin{array}{l}\text { The ERIM Report Series is distributed through the following platforms: } \\
\text { Academic Repository at Erasmus University (DEAR), DEAR ERIM Series Portal } \\
\text { Social Science Research Network (SSRN), SSRN ERIM Series Webpage } \\
\text { Research Papers in Economics (REPEC), REPEC ERIM Series Webpage }\end{array}$ \\
\hline Classifications & $\begin{array}{l}\text { The electronic versions of the papers in the ERIM report Series contain bibliographic metadata } \\
\text { by the following classification systems: } \\
\text { Library of Congress Classification, (LCC) LCC Webpage } \\
\text { Journal of Economic Literature, (JEL), JEL Webpage } \\
\text { ACM Computing Classification System CCS Webpage } \\
\text { Inspec Classification scheme (ICS), ICS Webpage }\end{array}$ \\
\hline
\end{tabular}




\section{SHOPPING CONTEXT AND CONSUMERS' MENTAL REPRESENTATION OF COMPLEX SHOPPING TRIP DECISION PROBLEMS}

BENEDICT G.C. DELLAERT, THEO A. ARENTZE, AND HARRY J.P. TIMMERMANS*

February 2008

* Benedict G.C. Dellaert is professor of Marketing, Department of Business Economics, School of Economics, Erasmus University, PO Box 1738, 3000 DR Rotterdam, The Netherlands, phone: +31 104081301 fax: +31 10408 9169, email: dellaert@few.eur.nl

Theo A. Arentze and Harry J.P Timmermans are associate professor and chaired professor respectively, Department of Urban Planning, Faculty of Architecture, Building and Planning, Eindhoven University of Technology, PO Box 513, 5600 MB Eindhoven, The Netherlands. Phone: +31 40 2472283; fax: + 3140 2475882; e-mail: t.a.arentze@bwk.tue.nl and eirass@bwk.tue.nl

\section{Acknowledgements}

Anouk den Hartog's research assistance in the data collection stage of this paper is greatly appreciated. 


\title{
SHOPPING CONTEXT AND CONSUMERS’ MENTAL REPRESENTATION OF COMPLEX SHOPPING TRIP DECISION PROBLEMS
}

\begin{abstract}
Depending on the shopping context, consumers may develop different mental representations of complex shopping trip decision problems to help them interpret the decision situation that they face and evaluate alternative courses of action. To investigate these mental representations and how they vary across contexts, the authors propose a causal network structure that allows for a formal representation of how context-specific benefits requirements affect consumers' evaluation of decision alternative attributes. They empirically test hypotheses derived from the framework, using data on consumers' mental representations of a complex shopping trip decision problem across four shopping contexts that differ in terms of opening hour restrictions and shopping purpose, and find support for the proposed structure and hypotheses.
\end{abstract}

Key words: Retailing, Consumer Decision-making, Mental Representations, Context Effects, Shopping Trip Decisions 
Consumer shopping trip decisions are complex in nature and typically require consumers to jointly choose from multiple shopping locations, particular stores, transportation options, and available time slots. Researchers have recognized the complexity of such decisions and have addressed complex shopping trip decision making in a variety of ways. Econometrically oriented research has expanded random utility consumer choice models to incorporate increasingly complex shopping trip decisions (e.g., Arentze, Oppewal, \& Timmermans 2005; Dellaert, Arentze, Bierlaire, Borgers, \& Timmermans 1998; Popkowski Leszczyc, Sinha, \& Sahgal 2004; Rhee \& Bell 2002). Behavioral researchers, in contrast, have challenged the assumptions of economic models and formulated approaches to account for non-normative effects in consumers' perceptions and evaluations of complex shopping trip alternatives (e.g., Brooks, Kaufmann, \& Lichtenstein 2004). While both research streams have analyzed consumer shopping decision making productively, they share a decision outcome oriented focus that has lessened emphasis on consumers' subjective construal of shopping trip decision problems (Beach \& Mitchell 1987; Johnson-Laird 2001; Loewenstein 2001; Weber \& Johnson 2006).

The objective of the current study is to provide further insights in this latter domain by investigating consumers' mental representations of complex shopping trip decision problems and how they may vary depending on the consumer's shopping context. Understanding variations in consumers' mental representations of shopping trip decision problems is crucial for researchers and managers in retailing, because different mental representations may lead to different attributes being incorporated in consumer decision processes, which in turn may lead to different consumer shopping trip choices. Thus, context-based variations in mental representations offer an additional - process-level - explanation for differences in evaluations and choices of shopping trip alternatives as reported in earlier research on shopping context effects (Mattson 1982; Van 
Kenhove, De Wulf \& Waterschoot 1999). Furthermore, understanding consumers’ mental representations of shopping trip decisions allows researchers and managers to better determine the appropriate attribute-level specification of choice models and choice experiments aimed at capturing consumer shopping trip behavior and how they need to vary by shopping context (Adamowicz et al. 2008). Thus, the accuracy of such models and experimental measurements can potentially be improved with these insights.

We develop a framework to describe how consumers, when faced with complex shopping trip decision problems, consciously or unconsciously construct simplified mental representations of the decision problem, enabling them to interpret the shopping context and to evaluate alternative courses of action. This process involves generating an individual, context-specific mental representation that captures the consumer's benefits requirements, attributes of the shopping trip decision alternatives (shopping location, transportation mode and shopping time slot), relevant context attributes, and the causal relationships between these attributes and benefits. Consumers can derive expectations about the conditions and consequences of their actions based on this mental representation (Johnson-Laird 2001). The proposed framework extends previous analyses of context-based differences in attribute activation for consumer shopping trip decisions in two important ways. First, rather than focusing on outcome-based shifts in attribute salience across shopping contexts (Mattson 1982; Van Kenhove, De Wulf \& Waterschoot 1999), the framework can capture shifts in consumers’ subjective construal of shopping trip decision problems that precede their evaluations of shopping trip alternatives (Loewenstein 2001). Second, by explicating the causal links between attributes and benefits that are activated in consumers' mental representation, the framework allows for a more insightful grouping and interpretation of differences in attribute activation in consumers’ mental 
representations based on the underlying benefits that consumers require. This latter type of interpretation is particularly helpful for researchers or managers to support them in deciding what subsets of attributes to select for choice models or choice experiments that are tailored to different shopping contexts.

On the basis of the proposed theoretical framework, we formulate specific hypotheses about the process by which shopping context affects consumers' incorporation of attributes in mental representations of shopping trip decision problems. We test the hypotheses with data on consumers' mental representations of a shopping trip decision problem across four shopping contexts that differ in terms of opening hours restrictions and shopping purpose. Data for these analyses were recorded in interviews with 120 respondents using a semi-structured interview protocol.

\section{THE IMPACT OF SHOPPING CONTEXT ON MENTAL REPRESENTATIONS OF COMPLEX SHOPPING TRIP DECISION PROBLEMS}

Complex shopping trip decision problems involve choices between courses of action in settings in which multiple choice opportunities exist, such as location, timing and transport mode, and outcomes may depend on many factors. At the basis of our analysis is the proposition that consumers' mental representations of such problems encompass a context-dependent selection of the characteristics of the decision alternatives and shopping context that they face as well as the consumer's needs that are relevant to the decision at hand. Though the notion that subjective perception is relevant for consumer behavior is a core assumption in consumer research that uses mental or cognitive mapping to investigate consumers' dispositions toward alternative actions (Atkins, Wood \& Rutgers 2002; Christensen \& Olson 2002), our approach is 
unique in that we focus on context-based variations in mental representations of the shopping trip decision problem and what these variations imply in terms of attributes that are most relevant to consumers’ shopping trip decisions. To distinguish conceptually between decision alternatives and context variables, we define context variables as "those factors particular to a time and place of observation, which do not follow from personal and stimulus [decision alternative] attributes, and which have a demonstrable and systematic effect on current behavior” (Belk 1974: 157).

Next, to describe mental representations in more detail, we distinguish between attributes and benefits (Myers 1976). Attributes relate to physically observable states of the system in which the consumer operates (e.g. opening hours, parking costs, etc.), while benefits describe the impact of these system states on the consumer's more fundamental physiological and psychological requirements for well-being (e.g., ease of traveling, convenience, etc.). Attributes and benefits are interrelated, because the value of an attribute to the consumer is determined by the benefit(s) to which it leads. Typically, there is a one-to-many relationship between benefits and attributes; a benefit is influenced by several attributes at the same time.

In complex decisions, a consumer's previous experiences provide a rich source of information for assessing and evaluating the likely consequences of alternative actions. By imagining possible conditions and alternatives in the context of a decision, the consumer is able to mentally simulate, on the basis of previous experiences, the outcomes of different alternative actions in terms of their resulting benefits. These mental simulations require effort which in turn imposes a constraint on the number and complexity of the causal relationships between attributes and benefits that the individual can evaluate. The more vivid the person's imagination, the better he or she is able to activate episodic memory, but in turn, the more mental effort he or she requires and, hence, the more restrictive the mental capacity constraint. 
This cognitive capacity restriction implies that constructing a mental representation for a given task requires trading off the power of the mental representation against working memory load (Johnson-Laird 2001). To be effective, activation of mental representation components is likely based on assessments of their power to discriminate between alternative actions that produce different evaluations. Thus, a key feature of mental representations is that they necessarily represent simplifications of the alternative actions and context and individual needs that are tailored to the decision. In particular, on the basis of meta-knowledge that a consumer activates, we expect that different benefits will be activated in consumer mental representations for different shopping contexts (Ratneshwar, Warlop, Mick, \& Seeger 1997; Warlop \& Ratneshwar 1993; Weber \& Johnson 2006).

By the nature of the causal relationships between attributes and benefits, the differences in benefit activation across shopping contexts also have implications for the activation of attributes. The reason is that conceptually different benefits are generated by different attributes (Myers 1976). Therefore, we expect the mental activation of attributes to depend on the benefits present in the mental representation of the shopping trip decision problem. Thus variation in benefit activation generates an indirect effect of shopping context on attribute activation (see Figure 1).

- INSERT FIGURE 1 ABOUT HERE -

Additionally, we expect that the effect of selective activation in mental representations may also lead to a second type of context-based activation shift in attributes. This second shift differs from the traditionally hypothesized effects of situation, which focus on shifts in required benefits (e.g., Gutman 1982; Srivastava, Leone, \& Shocker 1981). Though benefits and attributes 
are connected because different benefits require different attributes, we propose that, for a given benefit, the attributes that are most relevant to a decision may differ between contexts. The reason is that the impact of attributes on a given benefit may differ depending on context.

This suggested shift resembles previously suggested shifts in attribute-benefit connections that are based on differences between consumers, for example due to differences in consumer characteristics such as gender, age, income, etc. (Gutman 1982). Such variations in cognitive attribute-benefit connections have later been supported empirically in research comparing different spatial and cultural market segments, where it is was found that for food products, different segments connected different food attributes to the underlying benefit "good for health” (Ter Hofstede, Steenkamp \& Wedel 1999).

We extend these findings, by hypothesizing that differences in attribute-benefit relationships may also occur within a given consumer's mental representations. The reason is that due to shopping context variations the relevance of different attributes to generate a given benefit may vary, similarly to the way in which the relevance of different attributes may differ between different consumers. An example may further illustrate this principle. When faced with a shopping trip decision a consumer may always include the benefit of "feeling comfortable” in his or her mental representation of a shopping location decision. At the same time however, the consumer is likely to be aware that generating the benefit of "feeling comfortable" may or may not require the attribute "air-conditioned shopping environment," depending on the context variable "outdoor temperature." If the outdoor temperature is low, the consumer will not activate the attribute "air-conditioned shopping environment” as a feature leading to a more comfortable shopping experience. In case the outdoor temperature is very high however the attribute "airconditioned shopping environment” is highly relevant to feeling comfortable and it will be 
activated. Thus, attribute-benefit connections may be expected to shift depending on context. When we combine the existence of such shifts in the relevance of attributes in different contexts with the notion of limitations in working memory (Johnson-Laird 2001), we expect that the specific attribute activation in the mental representation across contexts may shift, even for the same set of activated benefits. Together with our earlier expectation of differences in attribute activation due to differences in benefit activation, this leads us to formulate the following hypothesis:

H1: Shopping context variations lead to differences in attribute activation in consumers' mental representations of a given complex shopping trip decision problem due to:

a. Differences in benefit activation that lead to differences in attribute activation, and b. Differences in attribute activation that occur even when controlling for differences in benefit activation.

The transition from the construction of the mental representation of the decision problem to its use as a basis for the consumer's choice occurs by evaluating decision alternatives in terms of how they perform on the activated benefit criteria, given the attributes of these alternatives and the context (McFadden 1986). Based on consumers' valuation of different benefits, and their specific choice rules, their benefit evaluations lead to a specific choice of action. Figure 2 depicts this evaluation structure, including the causal relationships between the different components in the mental representation.

- INSERT FIGURE 2 ABOUT HERE - 
Thus, attribute and benefit activations are instrumental to identifying which decision alternative provides the best match between generated and required benefits. Previous research on consumer decision-making rules has illustrated that under conditions of high dispersion of attribute level utilities, relatively simple lexicographic, or elimination-by-aspects type choice rules can be (almost) equally effective as normative weighted additive compensatory choice rules (Johnson \& Meyer 1984, Payne, Bettman \& Johnson 1988). These findings show that in principle no more attributes and benefits are required in a consumer's mental representation of a complex decision problem than are needed to select the most attractive decision alternative from a given set. Therefore, given individuals’ working memory constraints and the cognitive costs associated with mental effort, we anticipate that this type of simplification will indeed occur.

Since context variables can have an influence on the expected impact of attributes and benefits on the dispersion of consumer shopping utility across decision alternatives, this leads us to hypothesize that the number of attributes and benefits activated in working memory differs as a function of context effects on the utility dispersion of decision alternatives. In terms of the earlier example, if "feeling comfortable” is pursued as a desirable benefit and only very few of several shopping location alternatives considered provide an air-conditioned shopping environment on a hot summer day, then hardly any additional attributes may be needed in the mental representation to establish a preference between the few relevant shopping locations. If the outdoor temperature is low however, more attributes may be needed to single out the most attractive shopping location because the difference in utility between shopping location alternatives is much lower. Thus, if the context variable "outdoor temperature” is high, the impact of the attribute "air-conditioning” on feeling comfortable can be large enough for 
consumers to be confident that the shopping location preference will not change if only few additional attributes are taken into account, while for lower temperature levels several more attributes need to be taken into account. We hypothesize:

H2: Shopping context variations that increase the consequences of changes in attribute and benefit levels in terms of consumer shopping utility, lead to a lower number of activated attributes and benefits in consumers' mental representations of a given complex shopping trip decision problem.

Finally, it is important to note that although our theoretical framework describes how consumers represent a shopping trip decision problem rather than how they evaluate different decision alternatives, it also has important consequences for such evaluations. More specifically, consumers derive value from the benefits they experience (Srivastava, Leone, \& Shocker 1981) and achieve such benefit experiences when they experience a match between their benefit requirements and the benefits generated by the values of specific attributes. Therefore, consumer choices depend on their anticipation of the value of this match when they evaluate different decision alternatives (McFadden 1986). Differences in the activation of benefits and attributes in mental representations thus may influence evaluations of decision alternatives. Therefore, we also investigate this impact in our empirical analysis. 
EMPIRICAL ANALYSIS OF COMPLEX SHOPPING TRIP DECISION PROBLEMS: DATA COLLECTION AND ANALYSIS APPROACH

We conducted an experimental, empirical study to test the proposed framework and hypotheses in the context of consumer shopping trip decision problems. Each respondent in the study received a hypothetical shopping trip decision problem and articulated his or her considerations during a face-to-face interview. We varied the settings of the hypothetical decision problem over four shopping context conditions and assigned respondents randomly to a condition. The interview followed a semi-structured protocol (as we discuss subsequently), in which we recorded respondents' selection of shopping context and decision alternative attributes and benefits; the links among attributes, benefits, and decision variables; and respondents’ choices of actions. To focus our analysis on the attribute and benefit activation in the mental representations, we controlled for the decision alternatives in the shopping trip decision problem. Each interview resulted in a specification of the respondents' mental representation of the shopping trip decision problem, as well as their final choices. We next describe the sample, shopping trip decision problem and shopping context presented to respondents, interview protocol, and analysis approach.

\section{Sample}

The sample consisted of 120 university students from two university towns in the Netherlands. These students ranged in age from 18 to 35 years (average 22.9), 48.3 percent were women, and all were in possession of a drivers' license (a condition for participating in the experiment, which involved the choice of driving a car). Thus, the respondents constituted a relatively homogeneous group. For the purpose of this study, homogeneity is important to 
reduce possible differences in mental representations due to, for example, different sociodemographic background variables. A random sample very likely would have required a much larger sample size to observe the effects we report in the study. The reason is that a more heterogeneous sample would have generated additional noise in our observations, thus making it harder to test for differences between the mental representations in the different experimental contexts. The average total interview time per respondent was 55 minutes, and respondents received monetary compensation of 10 Euros for participating in the study. ${ }^{1}$ All respondents fully completed the interview after they had agreed to participate.

Three interviewers were trained to conduct interviews to elicit respondents’ mental representations of the hypothetical shopping trip decision problems using a semi-structured protocol. Respondents were randomly assigned to one of the four shopping context conditions, which were varied in the experiment and across interviewers. ${ }^{2}$

\section{Shopping trip decision problem presented to respondents}

Interviewers asked respondents to imagine they had very recently moved to a new town with which they were not familiar to start an internship. This context was realistic to our sample and emphasized the relative novelty of the decision to the respondent, so their mental representations would not simply reflect their existing habits. Respondents reported their considerations in the context of deciding on a shopping trip on a given working day. Respondents were told that a workday at their internship organization ran from 8.30 AM until 5.15 PM, with a flexibility of 15 minutes before and after work, and that they could take a lunch break of 45 minutes from 12:00-12.45 PM. In each scenario, respondents also learned that they 
needed their shopping items the next day; they could not postpone the shopping trip to a later date.

To describe the decision alternatives to the respondent, the interviewers presented them with a map of the hypothetical town (see Appendix A). The map distinguished and located three types of shopping alternatives: a major city center, a city-district center, and a neighborhood center. These types generally fit with the functional hierarchy of planned shopping centers in the Netherlands. To achieve generalizability across respondents, we also provided a general description of the three types of shopping centers to explain the types of stores typically available in each. Locations were chosen such that they represented feasible travel distances from home and work. The city center was located in between work and home, as was the citydistrict shopping center, whereas the neighborhood center was located close to the respondent's home. Thus, from his or her work or home location, the respondent could combine any of the three centers with a trip to or from work. Distances appeared separately on the map, in terms of average travel time for each of three modes of transport between the different destinations. Respondents were free to use a car, ${ }^{3}$ public transport by bus, or a bicycle.

To test the hypothesized effects of context variations, we independently varied two realistic shopping context factors: (1) Restricted vs. extended shopping opening hours, and (2) Type of product to be purchased (groceries vs. clothing). Restricted shopping hours implied that shops closed at 6:00 PM, whereas extended opening hours implied shops closed at 9:00 PM, in line with common practice in the Netherlands. Grocery shopping involved shopping for a required stock for half a week, with products classified according to daily groceries (bread, fruit, vegetables, meat, cheese, milk, soft drinks, and cookies) and other items (cleaning products) to allow respondents to assess the carrying capacity they would need for their transport mode. 
Clothing shopping involved the respondent needing a new item of clothing for a business presentation scheduled for the next day. Thus, in summary, the four shopping trip scenarios were as follows: (1) shopping for groceries on a day with restricted opening hours, (2) shopping for groceries on a day with extended opening hours, (3) shopping for clothing on a day with restricted opening hours, and (4) shopping for clothing on a day with extended opening hours.

We expect that in terms of the utility consequences of choices between different shopping trip alternatives (H2) the restriction of opening hours increases the utility differences between trip alternatives. The reason is that several trip options become more unattractive (due to total travel and shopping time constraints) when opening hours are restricted. For grocery vs. clothing shopping trips, we expect that the utility consequences of not achieving the stated clothing shopping objective are perceived by respondents as being more severe than those of not achieving the objective of buying the half-weekly grocery supply.

\section{Interview protocol}

We designed the protocol for the interviews to record the mental representations of the shopping trip decision problem for each respondent in a face-to-face setting. Structuring the interviews reduces interviewer and response errors and helps ensure consistency across scenarios. To ensure consistency further, we predefined the decision variables-timing of the shopping activity, whether to combine the working and shopping trip, choice of shopping location, and choice of transportation mode - in the interview materials and presented the same list to respondents in all four scenarios. In addition, we predefined an extensive list of shopping context and decision alternative attributes and benefits but did not show this list to the respondents. We based the decision variables and list of attributes and benefits on previous 
empirical research on consumer shopping location and transportation choices (Dellaert et al. 1998; Wen \& Koppelman 2000) and a pilot study conducted prior to the experiment.

To further warrant a correct classification of responses, interviewers were informed about the definitions of attribute and benefit variables and that attributes related to physically observable states of the system in which the consumer operates (e.g. opening hours, parking costs, etc.), while benefits described the impact of these system states on the consumer's more fundamental physiological and psychological requirements for well-being (e.g., ease of traveling, convenience, etc.). In the interview this distinction was supported by using predefined lists of variables for each of the two types, and by using different questions to the respondent to uncover attribute vs. benefit variables.

The pilot study consisted of two stages. In stage 1, we posed the scenarios, predefined decision variables, and attributes and benefits to 16 respondents during open interviews. The respondents critically evaluated the descriptions and brainstormed about possible omitted variables. On the basis of their responses, we refined the scenarios and variables and included them in the actual protocol. We then tested the protocol in stage 2 of the pilot study, in which we interviewed another 8 respondents. These respondents offered feedback about the clarity of the task and suggested any improvements to enhance clarity. We used these outcomes to refine the protocol and shorten the interview time.

In the main study, interviewers checked consistently to determine if a variable mentioned by the respondent was on the predefined list of variables and recorded the variable if a match occurred. During this process, the interviewers selected the variables but confirmed with the respondent if that selection was appropriate. When no suitable variable could be found on the list, the interviewer added the new variable, confirmed by the respondent. 
This semi-structured protocol draws on similar procedures used in cognitive mapping (Clarke \& Mackaness 2001; Eden 1988) and laddering interviews (Reynolds \& Gutman 1988). The procedure develops as follows: The decision variables are presented on printed cards, placed in a random arrangement on the table. The interviewer asks the respondent to place the cards in the sequence in which he or she prefers to deal with them, assuming he or she were to make decisions. Next, the interviewer goes through the list of decision variables in the order indicated by the respondent and, for each variable, informs the respondent about the decision alternatives and asks “What are your considerations when faced with these alternatives?” The interviewer identifies from the list of predefined attributes and benefits those that corresponded to the answer given (or adds the new attribute or benefit to the list). In any case, the interviewer verifies whether the respondent agrees with the classification and determines whether the attribute or benefit is causally linked to the alternative action variable. In case of doubts, these links are checked with the respondent.

Having identified the variable, the next step depends on the variable type. If the variable is an attribute, the interviewer proceeds by asking “Why is this variable influential in this case?” This "why” question generally results in an identification of an underlying benefit generated by the attribute, in which case no further "why" questions are needed. If another attribute is mentioned, the "why” question gets repeated until an underlying benefit emerges. When the originally mentioned variable is a benefit, the interviewer proceeds by asking "How is this variable influenced?” and this "how” question leads to the identification of other shopping context or alternative attributes. The interviewer also establishes causal links, depending on the type of variable, and verifies these links with the respondent if in doubt. The interviewer 
prompts other considerations by repeating this procedure until the respondent has no further considerations to mention.

After the first decision variable is processed, the interviewer repeats the entire procedure for the next decision variable, and so on, until all decision variables are processed. The same attribute or benefit may play a role in the context of multiple decision variables, because if a variable mentioned already occurred in the network, the interviewer does not add a new variable but rather establishes a link to the previously mentioned variable. Ultimately, this procedure leads to a completed representation of the attributes and benefits involved in the respondents' mental representation of the shopping trip decision problem, as well as the causal links among these attributes and benefits and the action variables involved in the decision.

Finally, after the mental representation is completed, the interviewer asks the respondent to select, for each decision variable, the alternative that he or she would choose in the given scenario. This choice is recorded for use in our empirical analysis as an indicator of consumers’ evaluations of different decision alternatives.

\section{Analysis}

At a general level, our three-part objective in the analysis is to test if (1) different benefits appearing in respondents' mental representations of shopping trip decision problems lead to the activation of different attributes in their mental representations (H1a); (2) different shopping contexts lead to the activation of different attributes in consumers' mental representations, even when we control for differences in benefit activation (H1b); and (3) the number of attributes and benefits activated in a consumers' mental representations is smaller for shopping contexts in which attributes and benefits have larger consequences (i.e., restricted opening hours scenarios 
and clothing shopping scenarios) (H2). Finally, to further validate our findings we also test if differences in attribute and benefit activation in mental representations do indeed lead to different evaluations of alternatives as expected.

We test H1a and H1b using the following methodological approach (for details, see Appendix B): The size of the influence of the presence of variables $Y$ on $Z$ (e.g., the effect of shopping context on benefit activation) equals the extent to which adding variables of type $Y$ increases the likelihood of correct predictions of variables $Z$. In other words, to measure the impact of benefit variables $B$ on activation of attribute variables $A$, we calculate the improvement achieved in predicting the activation of $A$ variables when we use information about the benefit variables $B$. If there is no impact of $B$ on $A$, information about $B$ does not lead to an improvement of predictions about $A$ in respondents' mental representations.

In testing H1b, we do not wish to evaluate the influence of $Y$ on $Z$ per se, but rather are interested in the additional influence of $Y$ on a third set of variables, $X$, while controlling for $Z$; that is, the influence of $Y$ on $X$ given $Z$. More specifically, we wish to test the impact of shopping context $S$ on attribute activation $A$, while controlling for the effect on $A$ of benefit activation $B$. We test this influence by calculating the improvement in predictions for the activation of $A$ variables when we add information about the shopping context $S$ to the information about benefits activation $B$ (see Appendix B, equation B.11).

Because we measure respondents' evaluation of alternatives as a binary response variable (i.e., chosen alternative equals 1 , all other alternatives equal 0 ), we analyze the effect of mental representation on evaluation using the same approach. In this case, we analyze the joint effect of benefit and attribute activation on the improvement in predicting consumers' choices of action alternatives. 
We use the log-likelihood as a measure of the goodness of fit of the predictions and test the significance of a difference in log-likelihood between predictions with and without the variables of interest. To do so, we use a rho-statistic that ranges from 0 to 1 and that represents the improvement of the prediction made by the model with the variable of interest relative to that of a model without the variable (see Appendix B, equation B.3). The impact of consumers’ mental representations on their evaluation of alternatives is tested using the same procedure.

To test $\mathrm{H} 2$ finally, we use a different approach and simply compare the frequencies with which different attributes and benefits appear in respondents' mental representations across the different shopping context conditions. We then test for significant differences using $t$-tests.

\section{RESULTS}

\section{Descriptive analyses}

As an initial example of the results obtained in the study, we offer Figure 3, which shows the mental representations of two respondents in the grocery and clothing shopping conditions. The decision variables appear in the top layer, attributes in the middle layer, and benefits in the bottom layer of the graph, and all arrows represent causal links. These two samples illustrate how mental representations may differ among persons and shopping contexts. For example, "width of assortment" was activated as an attribute in the mental representation of the respondent in the grocery shopping context, whereas “number of stores” served as an attribute in the mental representation of the respondent in the clothing shopping context. 
Tables 1 and 2 further show the observed frequency distributions across shopping contexts for attributes and benefits. ${ }^{4}$ In both tables, the chi-square values relate to the frequency distribution of each variable across the four scenarios. We find significant effects of shopping context on both the attributes and the benefits that are present in consumers' mental representations. Thus, though many attributes and benefits that are activated are identical across the different contexts, there are also a number of important differences. These results illustrate that the proposed approach is able to effectively support retail managers and researchers in selecting the most important components to include in their models and experiments.

At a more detailed level, and turning first to Table 1, the results indicate that though several of the most frequently mentioned attributes are constant across conditions (i.e., "simplicity of route”, "available time to shop”, “weather conditions”, and "total travel time”), context differences in attribute activation are also prominent. These context differences are relatively large between shopping trip types, such that a clear differentiation exists in the attributes for grocery versus clothing shopping trips. Thus, we find that product category in particular is an important context variable in consumers' mental representations of complex shopping trip decisions. For grocery shopping, "width of assortment”, "number of bags to carry”, “perishability of products”, "price level of assortment”, and “opening hours” are frequently present in the mental representation, whereas for clothing shopping, "number of stores", "flexibility of the departure time", "shopping atmosphere", and "familiarity with the location" are more frequently activated. Differences in the attributes that are activated between opening hour conditions are less pronounced.

With respect to benefits, Table 2 shows that benefit activation follows a similar pattern. There are several overlapping benefits that are frequently activated in all shopping contexts (i.e. 
“time saving”, "ease of traveling”, "reduces time pressure”, and "convenience of trip”), but there are also significant differences. In particular, "successful shopping” is activated more often for clothing shopping trips, whereas "quality of the merchandise", and "lower purchase costs" are more often activated for grocery shopping. Finally, we find that the benefit "flexibility of the trip" is mentioned more often if opening hours are restricted than if opening hours are extended.

\section{- INSERT TABLES 1 AND 2 ABOUT HERE -}

\section{Hypotheses tests}

We report the results of our analysis to test for the differential impact of benefits and shopping context on the attributes in mental representations (H1a, b) in Table 3. The analysis for H1a tests for the influence of the activation of different benefits on the activation of attributes. The results show that a significant improvement in the prediction of the presence of attributes is achieved if the benefits are known, in support of H1a. We also find support for an additional effect of shopping context on the activation of attributes when controlling for the activation of benefits in the mental representation. This finding provides support for H1b and demonstrates that in different shopping contexts, not only different benefits are activated, but also different attributes are required to define a given benefit. For example, the benefit "convenience of trip" may be determined by different attributes depending on if the context of the shopping trip involves grocery or clothing shopping. Jointly, the results pertaining to H1a and H1b indicate that the influence of shopping context on the mental representation of different attributes is substantial. 
To test H2, we calculate the sum of the attributes and benefits reported by respondents for each scenario and conduct one tailed $t$-tests for significant differences between scenario conditions. Across the two shopping categories, the differences in the number of variables for restricted and extended opening hours are in the expected direction, with fewer attributes and benefits for the (higher consequence) restricted opening hours condition, and are significant (16.0 vs. 17.1 variables, $p<0.05$ ). The reductions in number of variables are directionally supported but not significant when we investigate the differences separately at the level of attributes (9.6 vs. 10.2, $p<0.10$ ), and at the level of benefits (6.4 vs. 6.9, $p<0.10)$. We also compare the number of attributes and benefits activated in the grocery vs. clothing shopping trip scenarios. Here, we also find that in line with our expectation respondents activated significantly more variables in mental representations of the (lower consequence) grocery shopping trips than did those in the clothing shopping trip scenarios (17.6 vs. 15.6, $p<.01)$. This reduction again is similar at the levels of attributes (10.6 vs. 9.2, $p<0.01)$, as well as benefits (7.0 vs. $6.4, p<$ 0.05). If we look at a more detailed level, we find that although restricted opening hours significantly reduce the number of attributes across the two product categories, the reduction within the grocery shopping trip is not significant, while the reduction for the clothing shopping trip is strongly significant $(p<0.01)$. This latter finding indicates a stronger sensitivity to opening hours restrictions for clothing shopping trips than for grocery shopping trips. Jointly, these findings provide support for $\mathrm{H} 2$ in that the contexts in which choices between shopping trip alternatives have higher consequences resulted in the activation of fewer attributes and benefits in consumers’ mental representations of complex shopping trip decision problems.

Finally, to test the effect of the observed mental representations on explaining variations in consumers' choices of different shopping trip alternatives, we follow the analysis approach 
conducted for H1a,b (see Appendix B). To do so, we group together the effects of attributes and benefits to capture the total impact of knowing a person's mental representation in terms of loglikelihood improvement. In line with our expectations, we find a significant effect of mental representations on evaluations (Table 3).

\section{CONCLUSION AND DISCUSSION}

On the basis of our proposed theoretical structure for consumers' subjective construal of mental representations for shopping trip decision problems, we formulated two hypotheses. Our empirical analyses support these hypotheses, indicating that the impact of shopping context on the activation of attributes in consumers' mental representations depends both on the benefits that are activated in the specific context, and on how this context affects the consumers' cognitive connections between attributes and benefits (H1a, b). We also find that the number of benefits and attributes that is activated differs by shopping context depending on how the context shifts the utility consequences of alternative choices between shopping contexts (H2). Finally, we find support for our expectation that mental representations influence consumers' evaluations of decision alternatives.

At a more general level, the data and analyses support the claim that understanding consumers' mental representations is relevant for researchers and managers in retailing, because different mental representations lead to differences in shopping trip decision attributes that consumers take into account in their decisions, as well as to different evaluations of shopping trip alternatives. Thus, understanding the mental representation of the shopping trip decision problem can provide important cues to retail location managers with respect to the attributes they 
should use as a basis for analyzing consumer shopping decisions and for attracting consumers to their location.

\section{Managerial implications}

First, we note the potential implications of our findings for retail managers and researchers who use techniques such as choice modeling and conjoint analysis. We find that depending on consumers' mental representations different attributes may be most appropriate in models and hypothetical scenarios for consumers’ shopping trip alternatives. At a more detailed level, we also find that depending on the shopping context, different shopping trip attributes may be activated in the mental representation in connection with the same benefits. From a retail manager's perspective this implies that research statements about the benefits that consumers desire in shopping trip alternatives may not be sufficiently informative unless researchers also specify which attributes consumers mentally connect with these benefits in the specific shopping context at hand (e.g., a benefit such as shopping convenience may be defined by different location attributes depending on the type of product that is bought).

To further explore the managerial implications of our research, we also summarize our findings in terms of the sample respondents' evaluations of decision alternatives, as measured by their choice of alternatives. As was evident from our analysis (Table 3) these results are due -at least in part- to differences in mental representations. We find interesting differences in particular between grocery and clothing shopping contexts. In the case of grocery shopping, 15\% of the respondents chose to visit the neighborhood center, $38 \%$ chose to visit the regional shopping center, and $47 \%$ chose to visit the city center. In contrast, in the clothing shopping condition $100 \%$ of respondents chose to visit the city center location. Also, while most shopping 
trips are combined with the work trip, the number of combined trips is much higher for grocery shopping (97\%), than for clothing shopping (80\%), in which case a sizeable proportion of respondents prefer to make a separate shopping trip. For transportation mode choices finally, we find that in line with common practice amongst students in the Netherlands, most respondents chose to use a bicycle for their shopping trips ( $67 \%$ on average). Considerably more respondents however, chose to use the car to do their grocery shopping (38\%), than to go clothing shopping (23\%). Overall, few effects of opening hours conditions were observed.

We tentatively interpret these findings as an indication that consumers have a strong focus on efficiency considerations when planning their shopping trips for grocery shopping. For clothing shopping, efficiency appears to be also important, but considerations of being able to shop successfully weigh in more strongly. In other words, in the case of grocery shopping, the fit of a certain location in the route of conducting a wider activity chain seems to be consumers' primary concern, while attributes of the location itself are of relatively less concern in the choice of location. In contrast, in the case of clothing shopping, the choice of location appears to be primarily based on attributes of the location itself while the activity schedule (timing, tripchaining, and transport mode) is planned around the choice of clothing shopping location. Furthermore, the higher consequences of choosing "wrong” alternatives reduces the number of shopping trip attributes respondents considered when comparing between clothing shopping trip alternatives, especially when faced with a time constraint. This resulted in more homogenous location choices and a stronger adaptation of schedule choices (i.e., trip-chaining, timing, and transport mode) for clothing shopping than for grocery shopping. In contrast, grocery trip choices were stronger focused on efficiency, which lead to more homogenous route choices, 
with nearly all respondents combining the grocery shopping trip with the work to home trip, independent of time constraints.

This difference in attribute and (implicit) goal focus between grocery and clothing shopping also has implications for how shopping location choices may be affected differently by shifting consumer lifestyle patterns. Location choice may be of secondary nature for grocery shopping. This may imply for example that life-style shifts in society such as more individuals beginning to work from home (e.g., to avoid traffic jams in their daily commute), also lead to different grocery shopping location choices. For example, consumers may shift to grocery shopping locations that are close to home, and away from locations that are on the route from home to work. In contrast, clothing shopping location choices may be considered as a more stand-alone type of decision, which may be affected less by the consumer's working location. At the same time, releasing opening hours restrictions may affect clothing shopping trip decisions more strongly than grocery shopping decisions in terms of scheduling choices (timing, route and transport mode), because more flexible opening hours help alleviate the perceived risk of not succeeding in finding attractive clothing products in time.

\section{Theoretical implications}

The proposed theoretical framework recognizes that the decision situation in which a consumer operates has an effect on his or her behavior, in that he or she must reduce the complexity of the mental representation while maintaining the main causal relationships (Johnson-Laird 2001). This impact extends beyond the direct influence of situation on action outcomes (Belk 1974) and suggests that the influence of shopping context factors on choice behavior is greater than might be expected based solely on a consumer's causal knowledge and 
contextual preferences. Thus, a consumer's need to build simplified representations may amplify the effects of shopping context factors and the impact of context shifts in mental representations may appear as sudden and qualitative changes in the consumer's preferences for alternative shopping trip alternatives (e.g., when a decision problem is suddenly recast in another context).

To support this expected difference between the direct impact on preference of shopping context and its effect on mental representation, we conducted an additional analysis. In this analysis we first tested for a separate effect of shopping context on evaluation while controlling for differences in mental representation. This analysis captures previously demonstrated effects of context shifts in consumer preferences (e.g., Belk 1974), as might be the case when some shopping locations are more suitable for certain types of shopping occasions than others. In line with prior research, we find that shopping context alone also has a significant impact on evaluations, while controlling for mental representation (rho $=0.21, t$-value $=19.88, \mathrm{p}<0.05$ ).

To investigate the possibility that the mental representation results reported in Table 3 are driven only by the direct effect of shopping context on consumer preference, we also tested the effect of mental representation on evaluation when controlling for shopping context. We find this additional effect is significant as well (rho $=0.49, t$-value $=32.90, \mathrm{p}<0.05$ ), supporting the theoretical expectation that shopping context affects consumer evaluations both directly through context-based preference shifts, and indirectly through shifts in consumers’ mental representation of the shopping trip decision problem.

\section{Limitations and avenues future research}

Our study analyzed differences in mental representations of shopping trip decision problems in a static context, for a specific sample, and using hypothetical shopping trip 
decisions. Several authors however, emphasize the role of (long-term) memory in the subjective construal of decision situations (Beach \& Mitchell 1987; Weber \& Johnson 2006). Thus mental representations may change over time and based on a consumer's cumulative experiences. An interesting avenue for further research therefore might be to investigate how our theoretical framework and analysis approach might be used to study consumers in a more heterogeneous sample that make real-world shopping trip decisions. For example, it could be interesting to trace how new residents in a town adapt their mental representations of shopping trip decision problems over time (i.e, when they learn to shop in a their new town).

A potential practical limitation of our data collection approach is that it is relatively time consuming. Therefore, developing methods that could facilitate survey data collection of consumers' mental representations would be helpful. Despite the successful application of the interview protocol we developed for this study, considerable research effort could be saved if an automated computerized data collection could be implemented, such as, perhaps, the association pattern technique (APT) approach. The APT approach was developed to study relationships consumers perceive among different products, product benefits (e.g., low in calories), and their own personal objectives (e.g., to be healthy) and has been applied successfully to quantitative analyses of large-scale studies of product-benefit relationships (Ter Hofstede, Audenaert, Steenkamp, \& Wedel 1998). However, developing such an extension must consider how best to develop new survey formats that allow for a free-format elicitation of attributes and benefits in mental representation structures.

A related limitation of our findings is that although we took great care in defining attributes and benefits based on previous research and a qualitative pilot study and instructing our interviewers about the interpretation and applicability of these variables, the distinction 
between the two types of variables may sometimes be blurred, especially in the case of costrelated aspects. ${ }^{5}$ Thus, it would also be worthwhile to investigate in further detail to what extent attributes and benefits should be conceptually separated in the consumers' mental representation, or alternatively should be viewed as relatively similar cognitive constructs that vary on only one underlying dimension (e.g., level of abstraction), but still represent causally related features of decision alternatives.

In summary, our results demonstrate that mental representations have a strong and significant effect on consumer decisions and that research on mental representations can lead to a better understanding of consumer behavior. In turn, we hope our study offers a basis for further research on integrating mental representation effects in models and theories surrounding consumer decision making. 
APPENDIX A

MAP OF HYPOTHETICAL TOWN PRESENTED TO RESPONDENTS*

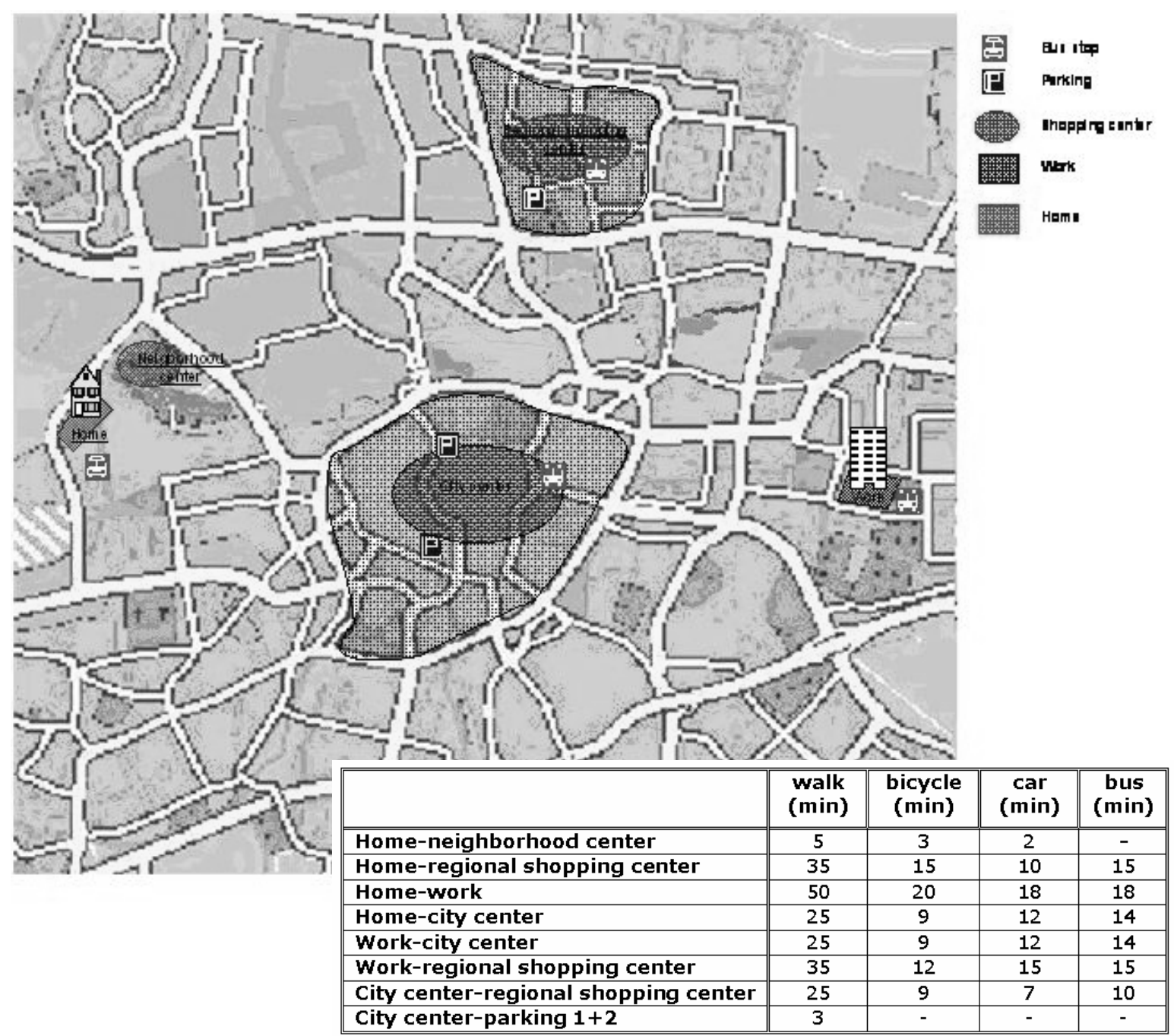

*The actual map used in the study was of higher resolution and in color and is available on request. 


\section{APPENDIX B}

\section{ANALYSIS APPROACH}

We capture the impact of the different variables in consumers' mental representations on the presence of other variables, as well as on consumers' choices, as follows: We define the size of the influence of the presence of variables $Y$ on $Z$ as the extent to which adding variables of type $Y$ to an existing model for predicting $Z$ increases the likelihood of correct predictions of variables $Z$. To capture this influence, we use a measure of likelihood of correct predictions, expressed as a log-likelihood statistic:

$$
L L(Z \mid Y)=\sum_{i=1}^{N} \sum_{z \in Z_{i}} \log \left[P\left(z \mid Y_{i}\right)\right]
$$

In this equation, $L L(Z \mid Y)$ is the log-likelihood of correctly predicting (the presence of) all $Z$ variables in the sample of cases, using the (presence of) $Y$ variables as predictors in each case; $N$ is the total number of cases; $Z_{i}$ and $Y_{i}$ are the sets of $Z$ variables (z) and $Y$ variables (y) observed in the network of case $i$; and $P\left(z \mid Y_{i}\right)$ is the predicted probability (of the presence) of $z$ occurring in the network of case $i$ if we have observed $Y_{i}$. When multiple $Y$ variables occur in the same network for a given $z$, for computational convenience, we conservatively use only one $y$, the best predictor, in our predictive models.

This log-likelihood (B.1) can be compared to the log-likelihood of correctly predicting $Z$ without observing the $Y$ variables:

$$
L L(Z)=\sum_{i=1}^{N} \sum_{z \in Z_{i}} \log [P(z)],
$$

where $P(z)$ is the predicted probability of $z$ being present in a case according to a model that uses no information about the case.

The improvement in prediction is represented with a common rho statistic defined as: 


$$
\rho(Z \mid Y)=\frac{L L(Z \mid Y)-L L(Z)}{L L(Z)},
$$

where $\rho(Z \mid Y)$ is the proposed rho-measure of the influence of (presence of) $Y$ on (the presence of) $Z$. For example, assume that we are interested in predicting the presence of the two attributes "time required for shopping" and "congestion on the road" and that we can use information about yes/no presence of the benefit "convenience of trip" in each case for which we want to make a prediction. Then, $L L(Z)$ represents the log-likelihood of correctly predicting the yes/no presence of each of these two attributes in each case if we do not use the information about yes/no presence of Convenience, $L L(Z \mid Y)$ represents the log-likelihood if we do use this information and $\rho(Z \mid Y)$ represents the improvement of the log-likelihood due to the use of the information. If "time required for shopping" and "congestion on the road" are often considered by individuals in relation to the benefit "convenience of trip", then knowing whether "convenience of trip" is included in the mental representation will increase the likelihood of correctly predicting the attributes and, consequently, the rho measure will be larger than zero.

In addition to the influence of $Y$ on $Z$, we also may be interested in the independent influence of a third set of variables, $X$, on $Z$-that is, the influence of $X$ on $Z$ given $Y$. We define the log-likelihood of a model extended with $X$ variables as follows:

$$
L L(Z \mid Y, X)=\sum_{i=1}^{N} \sum_{z \in Z_{i}} \log \left[P\left(Z \mid Y_{i}, X_{i}\right)\right],
$$

and the rho-measure of the independent influence of $X$ as

$$
\rho(Z \mid Y, X)=\frac{L L(Z \mid Y, X)-L L(Z \mid Y)}{L L(Z)} .
$$

As implied by this equation, the influence of $X$ on $Z$ given $Y$ is conceptualized as the further improvement in fit achieved by adding $X$ to the set of predictors relative to the null model. 
In terms of the earlier example, assume we are interested in the question whether an influence of the benefit "convenience of trip" on the activation of "time required for shopping” and "congestion on the road" is dependent on the context (i.e., scenario) variable grocery or clothing shopping. If it is, then knowing the shopping purpose in addition to knowing the presence of the benefit in each case would further increase our ability to predict the yes/no presence of these attributes. If it is not, then knowing the shopping purpose would not improve our predictions. In the test, $L L(Z \mid Y)$ represents the log-likelihood of correct predictions if we only use the benefit information, $L L(Z \mid Y, X)$ the log-likelihood if we in addition use the shopping purpose information and the rho measure would indicate the improvement of the prediction attributed to knowing the shopping purpose in addition to knowing the benefit activation.

This method can be applied to measure the specific effects in mental representations. To describe this approach, we introduce the following terms: Let $B_{i}$ be the set of benefits observed in case $i, A_{i}$ be the set of attributes observed in case $i, S_{i}$ be the shopping context (i.e., experimental condition) used in case $i$, and $U_{i}$ be the choice of alternatives observed in case $i$. Furthermore, say that we are interested in the following influences:

1. The influence of $S$ on $B$,

2. The influence of $A$ on $U$,

3. The influence of $S$ on $A$ given $B$, and

4. The influence of $S$ on $U$ given $A$.

To calculate the relevant rho-measures, we need models to generate predictions of the following kinds: $P(b), P(a), P\left(U_{i}\right), P\left(b \mid S_{i}\right), P\left(U_{i} \mid A_{i}\right), P\left(a \mid S_{i}, B_{i}\right)$, and $P\left(U_{i} \mid S_{i}, A_{i}\right)$, where $b \in B_{i}$ and $a \in A_{i}$ are specific network variables. We define the models as follows: 


$$
\begin{gathered}
P(b)=\frac{\text { freq }(b)}{N} \quad b \in B_{i} . \\
P(a)=\frac{\text { freq }(a)}{N} \quad a \in A_{i} . \\
P\left(U_{i}\right)=\frac{\text { freq }\left(U_{i}\right)}{N} . \\
P\left(b \mid S_{i}\right)=\frac{\text { freq }\left(b, S_{i}\right)}{\text { freq }\left(S_{i}\right)} \quad b \in B_{i} . \\
P\left(U_{i} \mid A_{i}\right)=\max _{a \in A_{i}}\left(\frac{\text { freq }\left(U_{i}, a\right)}{\text { freq }(a)}\right) . \\
P\left(U_{i} \mid S_{i}, A_{i}\right)=\max _{a \in A_{i}}\left(\frac{\text { freq }\left(U_{i}, S_{i}, a\right)}{f^{\prime} e q\left(S_{i}, a\right)}\right) . \\
P\left(a \mid S_{i}, B_{i}\right)=\max _{b \in B_{i}}\left(\frac{\text { freq }\left(a, S_{i}, b\right)}{\text { freq }\left(S_{i}, b\right)}\right) a \in A_{i} .
\end{gathered}
$$

In these equations, freq $(x)$ is the total number of times variable or value $x$ occurs in the sample of $N$ cases, freq $(x, y)$ is the total number of times $x$ and $y$ occur jointly in the sample, and so forth.

This method is complicated in that the number of predictors (e.g., presence of attributes and benefits) tends to be large compared with the number of observations (i.e., all cases). Therefore, we apply a k-fold cross-validation method to calculate a test statistic (Kohavi 1995). 
With this method, we draw one holdout set randomly from the sample in each of a specified number of rounds. We derive predictors from the remaining cases and used them to predict cases of the holdout set. The procedure is repeated $\mathrm{K}$ times, each time drawing the holdout set randomly from the total sample. The probability $p$ of holding a case out remains constant, so the test set has the same expected size in each cycle. The average rho-values across K draws represents an estimate of the true rho. The null hypothesis claims no improvement in fit, such that the mean rho is 0 . The t-statistic of difference between the mean and 0 is used to test the hypothesis. In the analyses, the parameters of the cross-validation method were set to $\mathrm{K}=100$ and $p=0.1$, in which case the number of degrees of freedom of the t-test equals $99(K-1)$. 


\section{REFERENCES}

Adamowicz, Wictor, David S. Bunch, Trudy-Anne Cameron, Benedict G.C. Dellaert, Michael Hanemann, Michael Keane, Jordan J. Louviere, Robert J. Meyer, Thomas Steenburgh, and Joffre Swait (2008). "Behavioral Frontiers in Choice Models,” Marketing Letters, forthcoming.

Arentze, Theo. A., Harmen Oppewal, and Harry J.P. Timmermans (2005). “A Multipurpose Shopping Trip Model to Assess Retail Agglomeration Effects,” Journal of Marketing Research, 42(1): 109-115.

Atkins, Paul W.B., Robert E. Wood, and Philip J. Rutgers (2002). “The Effects of Feedback Format on Dynamic Decision Making,” Organizational Behavior and Human Decision Processes, 88 (July): 587 - 604.

Beach, Lee Roy and Terence R. Mitchell (1987). “Image Theory: Principles, Goals and Plans in Decision Making,” Acta Psychologica, 66 (December): 201-220.

Belk, Russel W. (1974). “An Exploratory Assessment of Situational Effects in Buyer Behavior,” Journal of Marketing Research, 11 (2): 156-163.

Brooks, Charles M., Patrick J. Kaufmann, and Donald R. Lichtenstein (2004). “Travel Configuration on Consumer Trip-Chained Store Choice,” Journal of Consumer Research, 31 (2): 241-248.

Christensen, Glenn L. and Jerry C. Olsen (2002). “Mapping Consumers’ Mental Models with ZMET,” Psychology \& Marketing, 19 (6): 477-502.

Clarke, Ian and William Mackaness (2001). “Management ‘Intuition’: An Interpretative Account of Structure and Content of Decision Schemas Using Cognitive Maps,” Journal of Management Studies, 38 (2): 147-172. 
Dellaert, Benedict G.C., Theo A, Arentze, Michel Bierlaire, Aloys W.J. Borgers, and Harry J.P. Timmermans (1998). “Investigating Consumers' Tendency to Combine Multiple Shopping Purposes and Destinations,” Journal of Marketing Research, 35 (2): 177-188.

Eden, Colin, (1988). “Cognitive Mapping,” European Journal of Operational Research, 36 (1): $1-13$.

Gutman, Jonathan (1982). “A Means-End Model Based on Consumer Categorization Processes,” Journal of Marketing, 46 (2): 60-72.

Johnson, Eric J. and Robert J. Meyer (1984). "Compensatory Choice Models of Noncompensatory Processes: The Effect of Varying Context,” Journal of Consumer Research, 11 (1): 528-541.

Johnson-Laird, Philip N. (1983). Mental Models, Cambridge, MA: Harvard University Press. (2001). “Mental Models and Deduction,” Trends in Cognitive Sciences, 5 (10): 434-442.

Johnson-Laird, Philip N. (2001). "Mental Models and Deduction,” Trends in Cognitive Sciences 5 (10): 434-442.

Kohavi, Ron (1995). “A Study of Cross-Validation and Bootstrap for Accuracy Estimation and Model Selection,” pp 1137-1143 in Proceedings of the Fourteenth International Joint Conference on Artificial Intelligence (IJCAI). San Mateo, CA: Morgan Kaufmann.

Loewenstein, George (2001). "The Creative Destruction of Decision Research,” Journal of Consumer Research, 28 (3): 499-505.

Mattson, Bruce E. (1982). “Situational Influences on Store Choice,” Journal of Retailing, 58(3): 46-58.

McFadden, Daniel (1986). “The Choice Theory Approach to Market Research,” Marketing Science, 5 (4): 275-298. 
Myers, James H. (1976). "Benefit Structure Analysis: A New Tool for Product Planning.” Journal of Marketing, 40 (October): 23-32.

Payne, John W., James R. Bettman and Eric J. Johnson. (1988). “Adaptive Strategy Selection in Decision Making,” Journal of Experimental Psychology: Learning, Memory and Cognition 14 (3): 534552.

Popkowski Leszczyc, Peter T.L., Ashish Sinha, and Anna Sahgal (2004). "The Effect of MultiPurpose Shopping on Pricing and Location Strategy for Grocery Stores,” Journal of Retailing 80 (2): 85-99.

Ratneshwar, S., Luk Warlop, David G. Mick, and Gail Seeger (1997). "Benefit Salience and Consumers’ Selective Attention to Product Features,” International Journal of Research in Marketing, 14 (3): 245-259.

Reynolds, Thomas J. and Jonathan Gutman, (1988). "Laddering Theory, Method, Analysis, and Interpretation,” Journal of Advertising Research 28 (February-March): 11-31.

Rhee, Hongjai and David R. Bell (2002). "The Inter-Store Mobility of Supermarket Shoppers,” Journal of Retailing, 78(4): 225-237.

Srivastava, Rajendra K., Robert P. Leone, and Allan D. Shocker (1981). "Market Structure Analysis: Hierarchical Clustering of Products based on Substitution-In-Use,” Journal of Marketing, 45 (3): 38-48.

Ter Hofstede, Frenkel, Anke Audenaert, Jan-Benedict E.M. Steenkamp, and Michel Wedel (1998). “An Investigation into the Association Pattern Technique as a Quantitative Approach to Measuring Means-End Analysis," International Journal of Research in Marketing, 15 (1): 37-50. 
Ter Hofstede, Frenkel, Jan-Benedict E.M. Steenkamp, and Michel Wedel (1999), “International Market Segmentation Based on Consumer-Product Relations,” Journal of Marketing Research, 36(February), 1-17.

Van Kenhove, Patrick, Kristof de Wulf, and Walter van Waterschoot (1999). "The Impact of Task Definition on Store-Attribute Saliences and Store Choice,” Journal of Retailing, 75 (Spring): 125-37.

Weber, Elke U. and Eric J. Johnson (2006). “Constructing Preferences from Memory,” pp 397410 in The Construction of Preference. Sarah Lichtenstein and Paul Slovic (eds), New York, NY: Cambridge University Press.

Wen, Chieh-Hua and Frank S. Koppelman (2000). “A Conceptual and Methodological Framework for the Generation of Activity-Travel Patterns,” Transportation, 27 (1): 5-23. Warlop, Luk and S. Ratneshwar (1993). "The Role of Usage Context in Consumer Choice: A Problem Solving Perspective,” Advances in Consumer Research, 20 (1): 377-382. 


\section{FOOTNOTES}

${ }^{1}$ The interview also covered some shopping trip preference questions, asked after the interview protocol, which we do not analyze herein.

${ }^{2}$ In our analyses, we tested for potential interactions between interviewer and condition effects and found they were not significant. Therefore, we report the combined data from the three interviewers in the results section of the paper.

${ }^{3}$ Respondents that did not own a car were asked to imagine that they had borrowed one from their parents for the week.

${ }^{4}$ In total, we use 35 benefits and 94 attributes in the classifications to code the responses of respondents. We include only those variables whose total number of occurrences in the sample equalled at least 15 . Thus, we considerably reduce the degrees of freedom without a substantial loss of information. A sensitivity analysis reveals that results are robust across variation in the threshold over a wide range.

${ }^{5}$ We thank a reviewer for bringing this aspect to our attention. 
TABLE 1

Attributes Activated in Mental Representations**

\begin{tabular}{|c|c|c|c|c|c|}
\hline \multirow[b]{3}{*}{ Attribute } & \multicolumn{4}{|c|}{ Frequencies } & \multirow[b]{3}{*}{$\begin{array}{c}\text { Chi-Square } \\
\text { Value }\end{array}$} \\
\hline & \multicolumn{2}{|c|}{ Groceries } & \multicolumn{2}{|c|}{ Clothing } & \\
\hline & $\begin{array}{c}\text { Restricted } \\
\text { Opening } \\
\text { Hours }\end{array}$ & $\begin{array}{c}\text { Extended } \\
\text { Opening } \\
\text { Hours }\end{array}$ & $\begin{array}{c}\text { Restricted } \\
\text { Opening } \\
\text { Hours }\end{array}$ & $\begin{array}{c}\text { Extended } \\
\text { Opening } \\
\text { Hours }\end{array}$ & \\
\hline Simplicity of route & 26 & 28 & 25 & 22 & 0.81 \\
\hline Available time to shop & 13 & 20 & 23 & 25 & 7.18 \\
\hline Weather conditions & 13 & 16 & 13 & 19 & 2.01 \\
\hline Total travel time & 19 & 17 & 10 & 11 & 1.92 \\
\hline Number of stores & 5 & 3 & 23 & 23 & $34.75 *$ \\
\hline Width of assortment & 23 & 25 & 3 & 2 & $27.94 *$ \\
\hline $\begin{array}{l}\text { Time required to look for } \\
\text { parking }\end{array}$ & 14 & 9 & 16 & 10 & 4.74 \\
\hline Take a break from work & 9 & 13 & 7 & 14 & 2.48 \\
\hline $\begin{array}{l}\text { Flexibility of departure } \\
\text { time }\end{array}$ & 6 & 9 & 15 & 8 & $7.90 *$ \\
\hline $\begin{array}{l}\text { Time required for } \\
\text { shopping }\end{array}$ & 8 & 6 & 11 & 11 & 3.88 \\
\hline Number of bags to carry & 15 & 14 & 1 & 5 & $12.29 *$ \\
\hline Congestion on the road & 8 & 8 & 11 & 7 & 2.43 \\
\hline Accessibility of store & 11 & 8 & 8 & 3 & 3.94 \\
\hline Perishability of products & 11 & 19 & 0 & 0 & $28.59 *$ \\
\hline $\begin{array}{l}\text { Leisure time after } \\
\text { shopping }\end{array}$ & 10 & 7 & 3 & 9 & 2.98 \\
\hline Parking costs & 8 & 5 & 6 & 9 & 1.74 \\
\hline Storage capacity of vehicle & 12 & 9 & 2 & 4 & 6.85 \\
\hline Price level of assortment & 10 & 10 & 0 & 4 & $9.35^{*}$ \\
\hline Shopping atmosphere & 6 & 3 & 3 & 11 & $7.89 *$ \\
\hline Familiarity with location & 3 & 1 & 9 & 10 & $13.11^{*}$ \\
\hline Familiarity with stores & 6 & 6 & 3 & 5 & 0.50 \\
\hline Fuel cost when driving & 8 & 4 & 4 & 4 & 1.85 \\
\hline Opening hours & 10 & 9 & 0 & 0 & $16.01^{*}$ \\
\hline Requires physical activity & 4 & 9 & 3 & 2 & 4.87 \\
\hline $\begin{array}{l}\text { Travel time to and from } \\
\text { work }\end{array}$ & 4 & 5 & 3 & 6 & 0.97 \\
\hline $\begin{array}{l}\text { Travel time during lunch } \\
\text { break }\end{array}$ & 2 & 2 & 7 & 6 & 6.98 \\
\hline Crowdedness in stores & 2 & 7 & 3 & 4 & 2.80 \\
\hline Transportation costs & 3 & 4 & 2 & 7 & 3.59 \\
\hline
\end{tabular}

*Significant at $p<.05$.

** $N$ = 120; attributes mentioned fewer than 15 times are not included. 
TABLE 2

Benefits Activated in Mental Representations**

\begin{tabular}{|c|c|c|c|c|c|}
\hline \multirow[b]{3}{*}{ Benefit } & \multicolumn{4}{|c|}{ Frequencies } & \multirow[b]{3}{*}{$\begin{array}{l}\text { Chi-Square } \\
\text { Value }\end{array}$} \\
\hline & \multicolumn{2}{|c|}{ Groceries } & \multicolumn{2}{|c|}{ Clothing } & \\
\hline & $\begin{array}{c}\text { Restricted } \\
\text { Opening } \\
\text { Hours }\end{array}$ & $\begin{array}{c}\text { Extended } \\
\text { Opening } \\
\text { Hours }\end{array}$ & $\begin{array}{c}\text { Restricted } \\
\text { Opening } \\
\text { Hours }\end{array}$ & $\begin{array}{c}\text { Extended } \\
\text { Opening } \\
\text { Hours }\end{array}$ & \\
\hline Time saving & 27 & 30 & 26 & 26 & 0.63 \\
\hline Successful shopping & 18 & 15 & 24 & 29 & $6.76^{*}$ \\
\hline Ease of traveling & 22 & 22 & 12 & 14 & 3.20 \\
\hline Reduces time pressure & 16 & 16 & 20 & 17 & 2.18 \\
\hline Convenience of trip & 16 & 19 & 13 & 19 & 0.59 \\
\hline $\begin{array}{l}\text { Cognitive simplicity of } \\
\text { trip }\end{array}$ & 14 & 12 & 10 & 16 & 0.76 \\
\hline Promotes leisure time & 15 & 14 & 6 & 14 & 2.63 \\
\hline Reduces travel costs & 14 & 7 & 9 & 14 & 2.82 \\
\hline Quality of merchandise & 11 & 17 & 7 & 8 & $4.57 *$ \\
\hline Health-improving & 11 & 6 & 8 & 12 & 2.09 \\
\hline Flexibility of trip & 5 & 7 & 11 & 4 & $6.43^{*}$ \\
\hline Lower purchase costs & 8 & 9 & 0 & 3 & $9.53 *$ \\
\hline $\begin{array}{l}\text { Diversity of shopping } \\
\text { options }\end{array}$ & 4 & 5 & 6 & 4 & 1.23 \\
\hline Low effort shopping & 5 & 5 & 2 & 5 & 0.99 \\
\hline
\end{tabular}

*Significant at $p<.05$.

$* * N=120$; benefits mentioned fewer than 15 times are not included. 
TABLE 3

Mental Representation Effects: Attribute Activation and Decision Alternative Evaluation*

\begin{tabular}{lcc}
\hline & rho & t-Value \\
\hline $\begin{array}{l}\text { Impact of benefits and shopping context on attribute } \\
\text { activation }\end{array}$ & & \\
$\begin{array}{l}\text { Benefits activated } \rightarrow \text { Attributes activated (H1a) } \\
\text { Shopping context } \rightarrow \text { Attributes activated controlling for } \\
\text { benefits activated (H1b) }\end{array}$ & 0.33 & 108.44 \\
& & 25.64 \\
$\begin{array}{l}\text { Impact of mental representation on evaluation } \\
\text { Mental representation } \rightarrow \text { Decision alternative evaluation }\end{array}$ & 0.35 & 53.68 \\
\end{tabular}

$* \mathrm{~N}=120$, all values significant at $p<.05$. 
FIGURE 1

The Impact of Shopping Context on Benefit and Attribute Activation in Consumers’ Mental Representations of Complex Shopping Trip Decisions

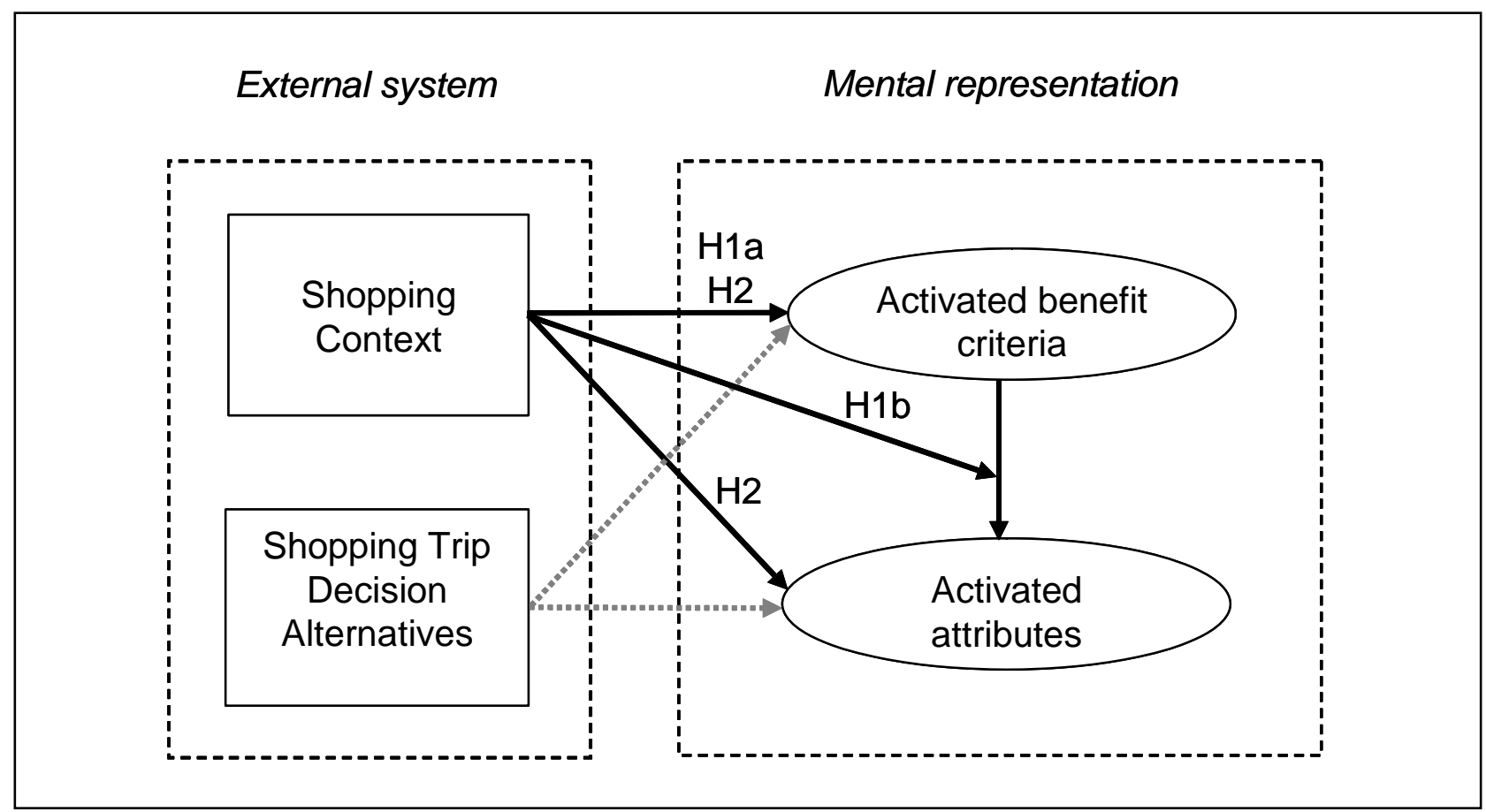


FIGURE 2

Causal Network Representation of Consumers' Evaluations of Complex Shopping Trip Decision Alternatives

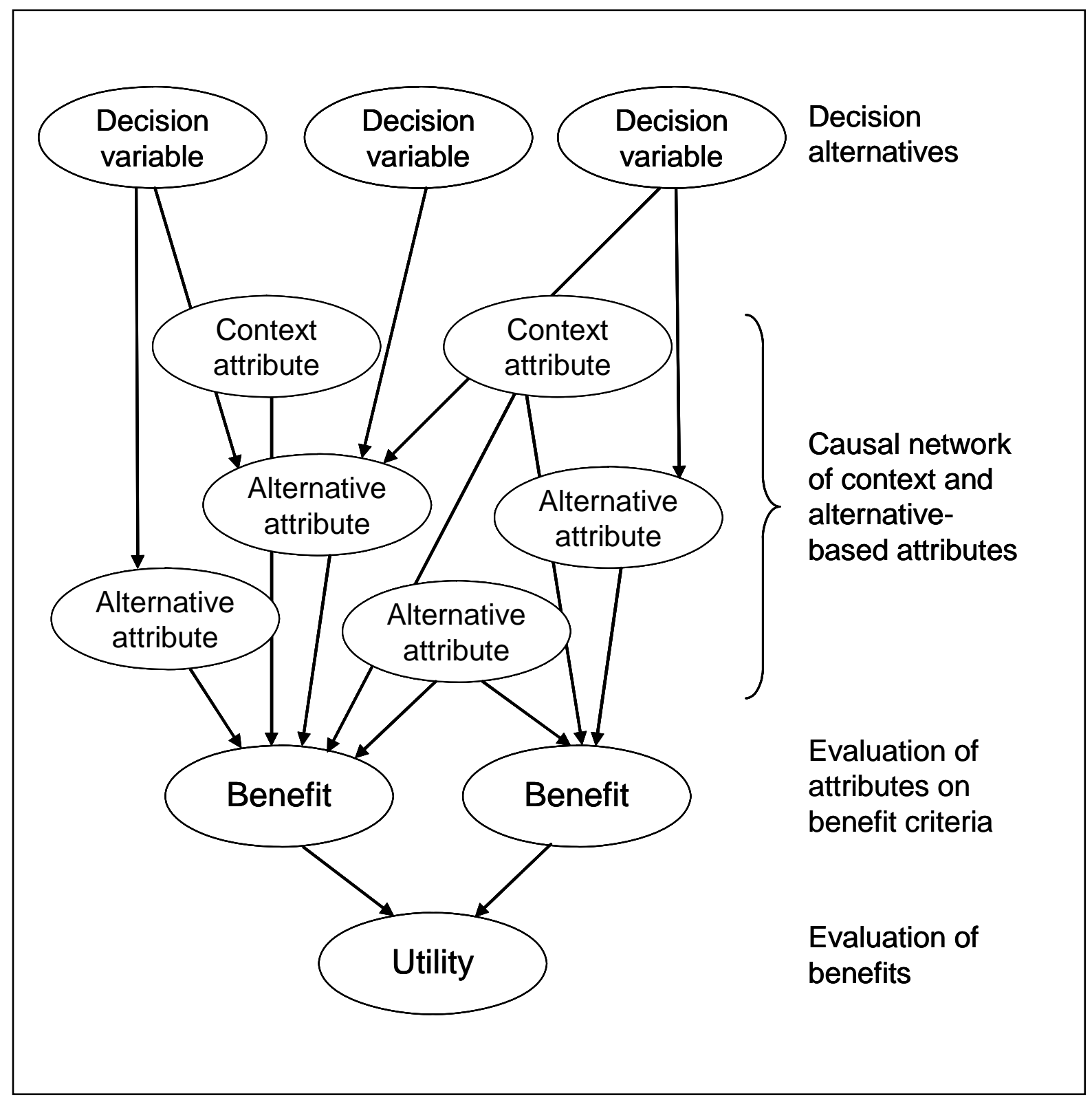


FIGURE 3

Example of Mental Representation Networks: Grocery and Clothing Shopping Contexts*

\section{Grocery}

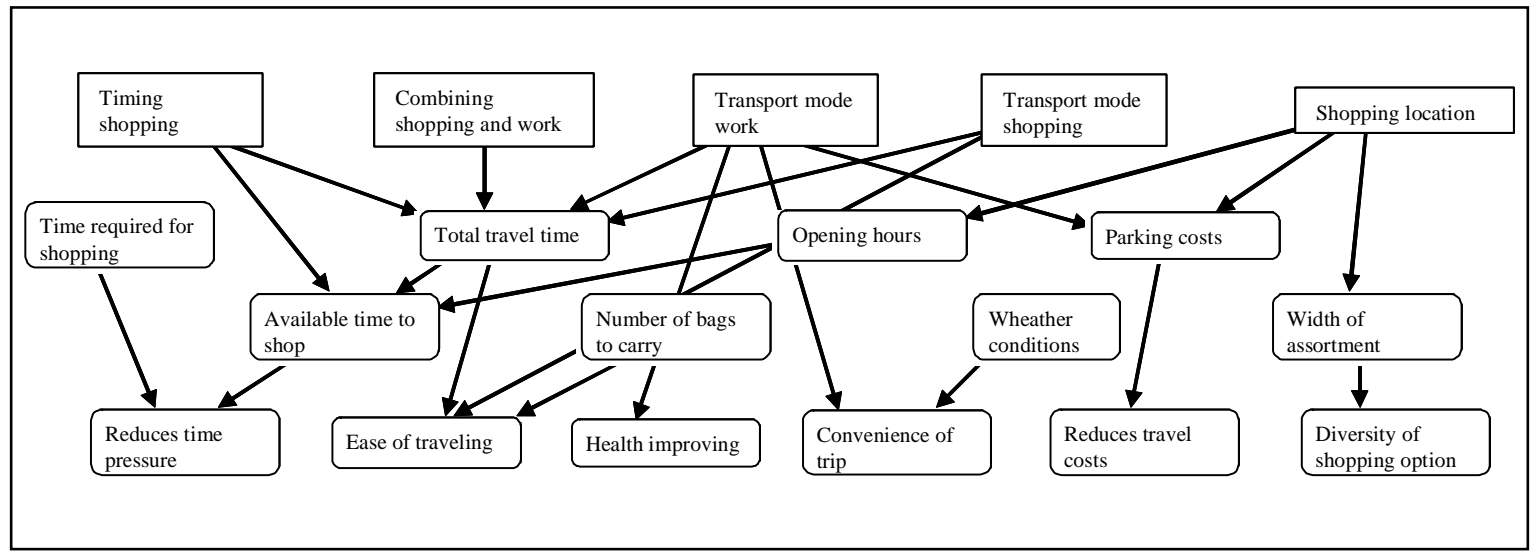

\section{Clothing}

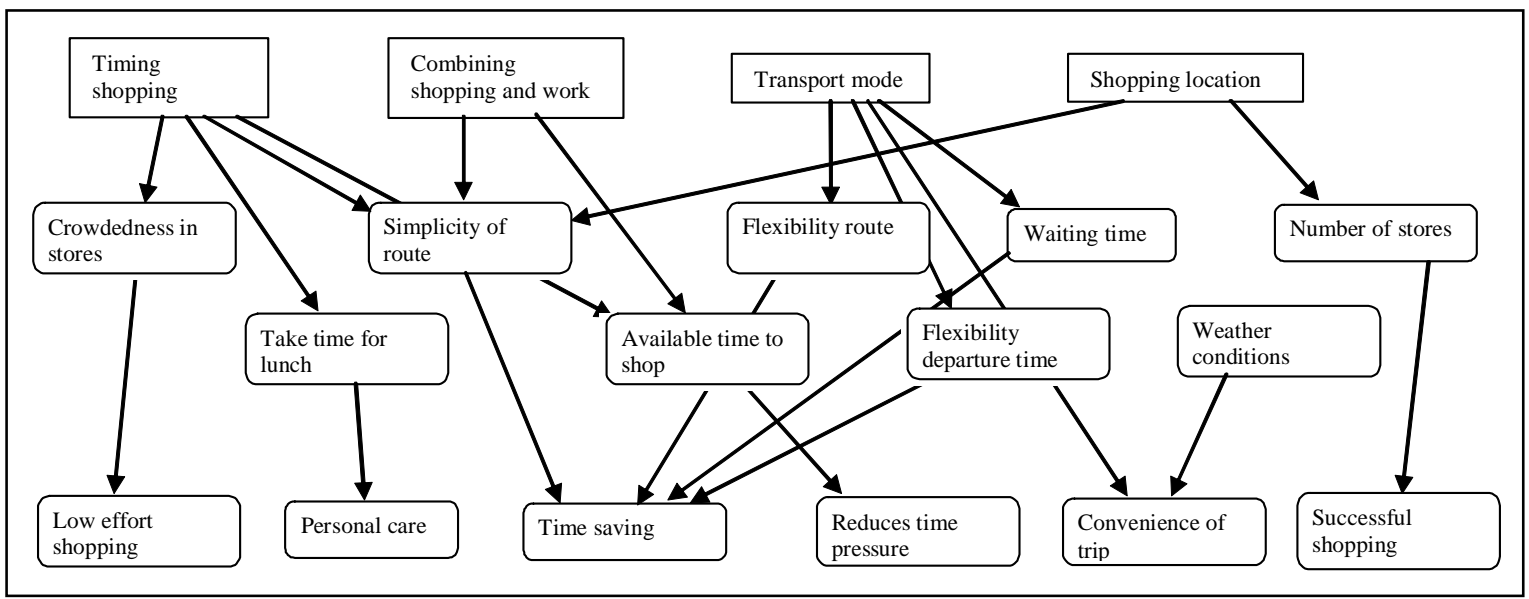

* Rectangular boxes represent decision variables; rounded boxes represent attributes and benefits mentioned by the respondent. The sequence of decision variables corresponds to the sequence indicated by the respondent. 


\section{Publications in the Report Series Research ${ }^{*}$ in Management}

\section{ERIM Research Program: "Marketing"}

2008

Experts' Stated Behavior

Youssef Boulaksil and Philip Hans Franses

ERS-2008-001-MKT

http://hdl.handle.net/1765/10900

The Value of Analogical Reasoning for the Design of Creative Sales Promotion Campaigns: A Case-Based Reasoning Approach

Niek A.P. Althuizen and Berend Wierenga

ERS-2008-006-MKT

http://hdl.handle.net/1765/11289

Shopping Context and Consumers' Mental Representation of Complex Shopping Trip Decision Problems Benedict G.C. Dellaert, Theo A. Arentze and Harry J.P. Timmermans

ERS-2008-016-MKT

http://hdl.handle.net/1765/11812

A complete overview of the ERIM Report Series Research in Management: https://ep.eur.nl/handle/1765/1

ERIM Research Programs:

LIS Business Processes, Logistics and Information Systems

ORG Organizing for Performance

MKT Marketing

F\&A Finance and Accounting

STR Strategy and Entrepreneurship 